OPEN ACCESS

Edited by: Emira Ayroldi,

University of Perugia, Italy

Reviewed by:

Carlo Chizzolini,

Université de Genève, Switzerland

Edith Janssen,

Janssen Research and Development,

United States

*Correspondence:

Tamarah D. de Jong

tamarahdesiree@gmail.com

Specialty section:

This article was submitted to

Autoimmune and Autoinflammatory

Disorders,

a section of the journal

Frontiers in Immunology

Received: 01 December 2018

Accepted: 08 April 2019

Published: 24 April 2019

Citation:

de Jong TD, Snoek T, Mantel E, van der Laken CJ, van Vollenhoven RF and Lems WF (2019) Dynamics of the

Type I Interferon Response During

Immunosuppressive Therapy in

Rheumatoid Arthritis.

Front. Immunol. 10:902.

doi: 10.3389/fimmu.2019.00902

\section{Dynamics of the Type I Interferon Response During Immunosuppressive Therapy in Rheumatoid Arthritis}

\author{
Tamarah D. de Jong *, Tanja Snoek, Elise Mantel, Conny J. van der Laken, \\ Ronald F. van Vollenhoven and Willem F. Lems
}

Amsterdam UMC, Vrije Universiteit Amsterdam, Rheumatology, Amsterdam Rheumatology and Immunology Center, Amsterdam, Netherlands

Objective: The type I interferon (IFN) response in rheumatoid arthritis (RA) has been extensively studied in relation to therapy with biological DMARDs (bDMARDs). However, the effect of conventional synthetic (cs)DMARDs and glucocorticoids (GCs) on IFN response gene (IRG) expression remains largely unknown, even though csDMARDS are used throughout all disease phases, including simultaneously with biologic therapy. This study was aimed to determine the dynamics of IFN response upon immunosuppressive treatment.

Methods: Whole blood was collected in PAXgene tubes from 35 RA patients who received either COBRA therapy (combination of prednisone, initially $60 \mathrm{mg}$, methotrexate and sulfasalazine) ( $n=14$ ) or COBRA-light therapy (prednisone, initially $30 \mathrm{mg}$, and methotrexate) $(n=21)$. Expression of $10 \mathrm{IRG}$ was determined by real-time PCR at baseline (T0), after 4 weeks (T4), and 13 weeks (T13) of treatment. IRG selection was based on the differential presence of transcription factor binding sites (TFBS), in order to study the therapy effect on different pathway components involved in IFN signaling.

Results: Seven of the 10 IRGs displayed significant changes during treatment $(p \leq$ 0.016). These 7 IRGs all displayed a particularly pronounced decrease between TO and T4 ( $\geq 1.6$-fold, $p \leq 0.0059$ ). The differences between IRG sensitivity to the treatment appeared related to the presence of TFBS for STAT1 and IRF proteins within the genes. The extent of the decreases between TO and T4 was similar for the COBRA- and COBRA-light-treated group, despite the differences in drug combination and doses in those groups. Between T4 and T13, however, IRG expression in the COBRA-light-treated group displayed a significant increase, whereas it remained stable or decreased even further in most COBRA-treated patients (comparison of mean fold changes, $p=0.011$ ). A significant association between IRG dynamics and clinical response to therapy was not detected.

Conclusions: Immunosuppressive treatment with csDMARDs, in this case a combination of prednisolone, methotrexate and sulfasalazine, substantially 
downregulates the IFN response in RA patients. The dynamics of this downregulation were partly dependent on the presence of TFBS within the IRGs and the combination and dosages of agents, but they were irrespective of the clinical response to therapy.

Keywords: rheumatoid arthritis, interferon, interferon response, biomarker, immunosuppression

\section{INTRODUCTION}

Early treatment of rheumatoid arthritis (RA) has proven effective in decreasing disease activity and limiting joint damage $(1,2)$. One treatment strategy which has shown effectiveness in early RA is COBRA (Dutch acronym for COmbinatietherapy Bij Reumatoïde Arthritis), which is a step-down strategy consisting of initial high dose prednisolone (60 mg per day), methotrexate (MTX) and sulfasalazine (SSZ). Due to rheumatologists' concerns with respect to the high initial prednisolone dose and the complexity of the drug schedule, COBRA-light strategy was introduced, which consists of a lower initial prednisolone dose (30 mg/day), combined with increasing doses of MTX (10-25 mg in 9 weeks) and no SSZ. The two strategies have shown to be similarly effective (3-5).

The use of glucocorticoids (GCs) such as prednisolone and conventional synthetic disease-modifying anti-rheumatic drugs (csDMARDs) such as MTX and SSZ is not restricted to early disease. In fact, these therapies are used throughout all phases of the disease, either as monotherapy or in combination, including simultaneously with biologic therapy (6).

With regard to biologic therapy, we have previously demonstrated that the predictive performance of the type I interferon (IFN) response gene set for non-response to rituximab was impaired when patients were using prednisolone at the moment of blood collection (7). Besides rituximab, IFN response gene (IRG) expression has also been described as a predictor for other bDMARDS such as anti-TNF agents and tocilizumab, and RA onset (8-11).

However, studies on the potential influence of csDMARD and GC (co-)medication yet remain scarce. Insight into the effect of these therapies on the IFN response, as well as the potential relation between IRG expression and the clinical response to csDMARD and GC therapy, are highly relevant in order to further understand the role of the IFN response in RA.

In vitro studies have shown that GC signaling could inhibit type I IFN signaling by competition for the same intracellular signaling components, i.e., the IFN regulatory factors (IRFs) $(12,13)$ and by inhibition of the transcription factor STAT1 (14). Accordingly, we have observed that RA patients who were treated with the GC prednisolone indeed displayed lower IRG expression compared to patients who had not received this treatment $(7,15)$. Although this decrease was not observed with methotrexate (MTX) use and appeared dependent on prednisolone dose, a causal relation could not be established due to the cross-sectional nature of the study. Moreover, since the study was performed in patients who were about to start on biologic therapy, hence who no longer benefitted from the csDMARD and GC therapies, an analysis in relation to clinical response to these therapies could not be made. The present study was focused on exploration of the IFN response during COBRA and COBRA-LIGHT therapy in RA. The sample collection within the COBRA and COBRAlight cohorts enabled us to investigate this in a longitudinal manner and additionally examine the potential relation with clinical response.

\section{METHODS}

\section{Patients and Treatment}

All patients in the current study participated in the COBRAlight study, a randomized, open, multicenter trial comparing two treatment schedules for the treatment of early RA (http:// www.controlled-trials.com; ISRCTN55552928). Details of that study have been reported previously (3). In short, DMARDnaïve Dutch patients with recent-onset RA according to the 1987 revised American College of Rheumatology criteria (16) were included and randomized to the COBRA-light or COBRA strategy. Whereas, COBRA therapy consists of initially high-dose prednisolone (60 $\mathrm{mg} /$ day) combined with sulfasalazine (SSZ) and low-dose methotrexate (MTX) (7.5 mg/week), COBRAlight consists of a lower initial prednisolone dose $(30 \mathrm{mg} /$ day $)$ but a higher starting dose of MTX (10 mg/week) and no SSZ.

For this study, 36 patients were selected based on availability of PAXgene tubes at baseline (T0), after 4 weeks (T4) and 13 weeks (T13) at the Amsterdam Rheumatology and Immunology Center, location Reade, Amsterdam, The Netherlands. Fifteen patients received COBRA therapy and 21 patients received COBRA-light therapy. Therapy response was defined as a Disease Activity Score in 44 joints (DAS) $\leq 2.4$ after 26 weeks of treatment. Additionally, the change in DAS ( $\triangle \mathrm{DAS})$ after 13 weeks and 26 weeks was also assessed.

This study was approved by the medical ethics committee of VU University Medical Center and Reade, Amsterdam, The Netherlands, and informed consent was obtained from all donors.

\section{RNA Isolation and cDNA Synthesis}

From each donor, blood was collected into a PAXgene tube (PreAnalytiX $\mathrm{GmbH}$ ) at baseline and after 4 weeks and 13 weeks of treatment. The PAXgene tubes were stored at $-20^{\circ} \mathrm{C}$ until further processing. After overnight thawing at room temperature, total RNA was isolated using the PAXgene Blood RNA kit (PreAnalytiX GmbH) according to the manufacturer's instructions. Total RNA concentration was measured using the Nanodrop spectrophotometer (ThermoFisher Scientific Inc.). From each sample, 250 ng RNA was reverse-transcribed into cDNA using a Revertaid H-minus cDNA synthesis kit (ThermoFisher Scientific Inc.). 
TABLE 1 | IFN response gene selection.

\begin{tabular}{|c|c|c|c|c|c|}
\hline \multirow[t]{2}{*}{ Genes } & \multicolumn{4}{|c|}{ Transcription factor binding sites } & \multirow{2}{*}{$\begin{array}{l}\text { Reason for } \\
\text { selection }\end{array}$} \\
\hline & IRF proteins & STAT1 & STAT3 & $N F_{K} B$ & \\
\hline \multicolumn{6}{|c|}{ INITIAL GENE SELECTION } \\
\hline IFI44L & IRF7, IRF8 & $\mathrm{x}$ & $x$ & - & Technical control \\
\hline IFI6 & IRF7, IRF8, ISRE & - & - & - & IRF-specific \\
\hline IFITM1 & - & - & - & $x$ & NFкB-specific \\
\hline IL1RN & - & - & $x$ & - & STAT-specific \\
\hline$M \times 1$ & IRF7, ISRE & $x$ & $x$ & - & Technical control \\
\hline RSAD2 & IRF7 & - & $x$ & - & Technical control \\
\hline \multicolumn{6}{|c|}{ ADDITIONAL SELECTION } \\
\hline HERC5 & IRF7, ISRE & - & - & - & IRF-specific \\
\hline IFITM2 & - & - & $x$ & $x$ & $\begin{array}{l}\text { IRF- and } \\
\text { STAT1-lacking }\end{array}$ \\
\hline LYGE & - & $x$ & $x$ & $x$ & IRF-lacking \\
\hline SERPING1 & - & $x$ & $x$ & $\mathrm{x}$ & IRF-lacking \\
\hline
\end{tabular}

IRGs that contained a binding site for only one type of transcription factor were selected. Additionally, three other genes were included as technical controls.

" $X$ " indicates that the gene contains a binding site for that transcription factor, whereas "-" indicates absence of the TFBS in that gene. ISRE; IFN stimulated response element, binding site of the ISGF3 complex which consists of STAT1, STAT2, and IRF9. Binding is IRF9-dependent, hence this is considered an IRF-specific binding site.

\section{Interferon Response Gene Selection and Real-Time PCR}

Because GCs have been demonstrated to inhibit the IFN response in vitro via interaction with specific signaling components such as IFN regulatory factors (IRFs) $(12,13)$ and STAT1 protein (14), three IFN response genes (IRGs) were selected for the presence of specific transcription factor binding sites (TFBS). Thereto, all 45 IRGs that were previously described to be part of the IFN signature in RA (17), were submitted to the Transfac algorithm available from Interferome (http://interferome.its.monash.edu. au), an online database of IRGs (18). As shown in Table 1, IL1RN only contained a binding site for the transcription factors STAT3, IFITM1 only for NFKB and IFI6 only for IRF-proteins, such as IRF7, IRF8, and IRF9, which binds the IFN responsive element (ISRE). In addition, RSAD2, MX1, and IFI44L were taken along as positive controls because of their known well-detectability $(9,15)$. To confirm our initial observations, four additional genes were included based on the presence of certain TFBS (see Table 1). Real-time PCR was performed using Taqman gene expression assays and ABI Prism 7500 HT Sequence Detection System (Thermo Fisher Scientific Inc.), according to the manufacturer's protocols. Gene expression values were calculated relative to a standard curve and normalized to the average expression of two housekeeping genes: $18 S$ rRNA and HPRT.

\section{Statistical Analysis}

One patient was not included in the analyses as the RNA yield of its T4 sample was not sufficient for further measurements. Statistical analyses were performed using IBM SPSS Statistics 22. Data normality was checked according to Shapiro-Wilk test, with a normal distribution if $p>0.05$. Because most data
TABLE 2 | Cohort characteristics of the COBRA and COBRA-light groups.

\begin{tabular}{lccc}
\hline & All patients & $\begin{array}{c}\text { COBRA group } \\
\text { COBRA-light } \\
\text { group }\end{array}$ & $\begin{array}{c}\text { Coup } \\
N\end{array}$ \\
\hline Age, years, median (IQR) & $54(45-60)$ & $56(44-61)$ & $54(45-59)$ \\
Female gender, $n(\%)$ & $25(71)$ & $9(64)$ & $16(76)$ \\
DAS at baseline, median (IQR) & $4.0(3.3-4.6)$ & $4.0(3.7-4.6)$ & $4.0(3.3-4.5)$ \\
DAS at T26, median (IQR) & $1.7(0.8-2.1)$ & $1.2(0.4-2.0)$ & $1.8(1.0-2.4)$ \\
DAS at T26 $\leq 2.4, n(\%)$ & $28(80)$ & $12(86)$ & $16(76)$ \\
\hline
\end{tabular}

$I Q R$, interquartile range.

were not normally distributed, non-parametric tests were used for most comparisons. Longitudinal changes in IRG expression during treatment were tested using Friedman tests, followed by Wilcoxon signed ranks test. The comparisons of COBRA and COBRA-light therapy and responders and non-responders were performed using Mann-Whitney U test. Correlations between IRG expression and $\triangle \mathrm{DAS}$ were assessed using Spearman correlation and correlations between IRG expression and ${ }^{2} \log$ transformed CRP and ESR ratios were assessed using Pearson correlation. $P<0.05$ were considered statistically significant.

\section{RESULTS}

\section{Patient Characteristics}

Demographic and clinical data are shown in Table 2. No significant differences were observed in clinical characteristics between the COBRA and the COBRA-light group. After 26 weeks, the COBRA-light group displayed a higher DAS value and a lower percentage of patients with DAS values below 2.4. However, these differences did not reach significance, neither at later time points (data not shown, $p \geq 0.45$ ), which is in line with previously demonstrated non-inferiority of COBRA-light versus COBRA therapy (3-5).

\section{Dynamics of the IFN Response During Immunosuppressive Therapy}

In order to gain insight into the dynamics of the IFN response during COBRA and COBRA-light therapy, we first analyzed the expression of 6 IRGs at baseline (T0) and after 4 weeks (T4) and 13 weeks (T13) of treatment in the complete group of COBRA and COBRA-light combined.

As shown Figure 1 and Supplementary Table 1, expression of all measured IRGs except IFITM1 and IL1RN displayed significant changes over all time points (Friedman test, $p \leq$ 0.016 , vs. $p \geq 0.057$ for IFITM1 and ILIRN). These changes were most pronounced at $\mathrm{T} 4$, with median fold changes ranging from only 1.1-fold and 1.3-fold for IFITM1 and ILIRN, respectively, up to 2.5-fold for RSAD2 (Supplementary Figure 1A). In the significant genes, i.e., IFI6, IFI44L, MX1, and RSAD2, 69-77\% of patients displayed a more than 1.2 -fold decrease, whereas only 46 and $57 \%$ of the patients showed a more than 1.2-fold decrease in IFITM1 and ILIRN, respectively (Supplementary Table 1). As displayed in Supplementary Figure 2, the extent of the fold 


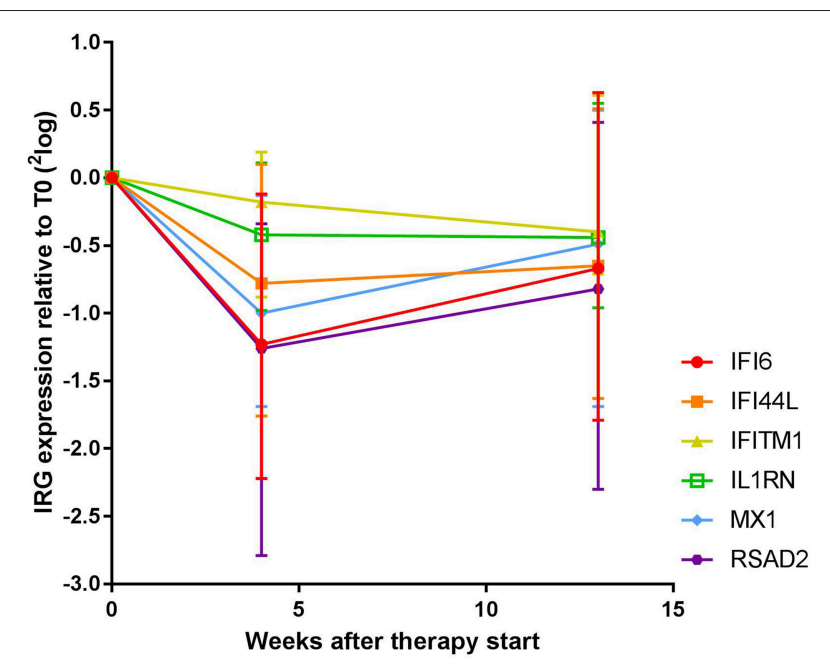

FIGURE 1 | Expression dynamics of individual IRGs during COBRA and COBRA-light therapy. Both cohorts were merged for initial analysis.

change of T4 and T0 was partly dependent on the gene expression levels at baseline, i.e., higher baseline expression generally led to higher fold decreases. However, several patients displayed relatively low baseline expression and relatively high fold changes and vice versa, indicating that the extent of the fold change could not be fully explained by the baseline expression values.

Between T4 and T13, changes in IRG expression were either non-significant or displayed a moderate increase at the group level (1.0 to 1.4 -fold increase, $p=0.012-$ 0.29 ), indicating stabilization or even reversal of the IRG decrease that occurred after 4 weeks of treatment. Of note, overall dynamics were largely variable between patients (see Supplementary Figure 1B). Individual dynamics over time are displayed in Supplementary Figure 3.

\section{Relation Between Transcription Factor Binding Sites and Sensitivity to Immunosuppressive Downregulation}

Remarkably, the two genes that appeared least affected by the COBRA and COBRA-light therapy, IFITM1 and ILIRN, both lacked binding sites for IRF-transcription factors and STAT1 (see Table 1). This implies that the therapy-related IRG reduction might be IRF-dependent and/or STAT1-dependent. In order to test this hypothesis, an additional selection of IRGs was made, based on the presence of binding sites for either IRF or STAT1 (see Table 1). As shown in Figure 2 and Supplementary Table 2, the additional IRG that lacked a TFBS for IRF proteins or STAT1, i.e., IFITM2 displayed only moderate changes upon treatment, similar to IL1RN and IFITM1 $(p=0.49)$. Accordingly, the additional genes with a TFBS for IRF proteins and/or STAT1 showed a considerable downregulation at the group level $(p$ $\leq 0.012$ ). This further suggests that the therapy-related IRG reduction is largely IRF- and STAT1-dependent.

\section{Differences in Dynamics of IFN Response Between COBRA and COBRA-Light Therapy}

Since the main difference between COBRA and COBRA-light therapy is the dose of prednisolone and the use of SSZ, and previous studies have shown a potential suppressing effect of those two agents on IRG expression $(7,13,15)$, we next analyzed the two therapy groups separately. The 7 IRGs with most distinct dynamics over time (HERC5, IFI6, IFI44L, LY6E, MX1, RSAD2, and SERPING1) were highly correlating (Spearman $r \geq 0.53, p<$ 0.001 ), hence expression levels of these genes were averaged into a 7-IRG score for visualization purposes.

As shown in Figure 3, both the COBRA and the COBRA-light group displayed a similar median decrease in IRG expression between T0 and T4, despite the difference in prednisolone dose and SSZ use (Comparison of fold changes, $p \geq 0.19$ ). However, IRG dynamics between T4 and T13 appeared strikingly different; whereas in the COBRA-treated group IRG expression displayed only minor changes (median 1.1-fold, maximum 1.6fold increase), the majority of the patients in the COBRA-lighttreated group displayed an increase in expression (median 1.8fold, up to maximum 9.9-fold.Comparison of fold changes in 7-IRG score $p=0.029$ ). Significantly more COBRA-light-treated patients displayed an increase of at least 1.2-fold (chi-square $p$ $=0.019$ ). Similar results were found for the individual IRGs (Supplementary Figure 4). There was no significant correlation between T13/T4 ratio and baseline IRG expression in these groups ( $p \geq 0.12$, data not shown), indicating that these dynamics are dependent on the treatment rather than on the baseline expression levels.

\section{Dynamics of IFN Response in Relation to Clinical Response to Therapy}

Despite the significant changes in the IFN response observed at the group level, we also observed substantial variation in IRG expression between individuals. For example, some patients did not display downregulation in any of the IRGs between T0 and $\mathrm{T} 4$, or only in a part of them (data not shown). Therefore, we also investigated whether these inter-individual variations could be related to the clinical response to COBRA and COBRAlight therapy.

Non-response was defined as DAS $>2.4$ at T26. As such, the merged cohort consisted of 7 non-responders and 28 responders. Due to low numbers, the two cohorts could not be analyzed separately. In line with previous reports, no correlation was observed between baseline DAS and IRG expression $(15,17)$ (data not shown). As shown in Table 3 and Supplementary Table 3, no significant differences in the 7-IRG score or any of the treatment-sensitive IRGs were observed between responders and non-responders, at baseline nor in the expression and dynamics after 4 and 13 weeks $(p \geq 0.059$ ). Furthermore, no significant correlation was observed between IRG expression and dynamics and the change in DAS after 13 and 26 weeks (unadjusted $p$-values $\geq 0.045$ ).

At T4, where the maximum IRG decline was observed, DAS was not determined. Instead, we investigated CRP and ESR at T4 


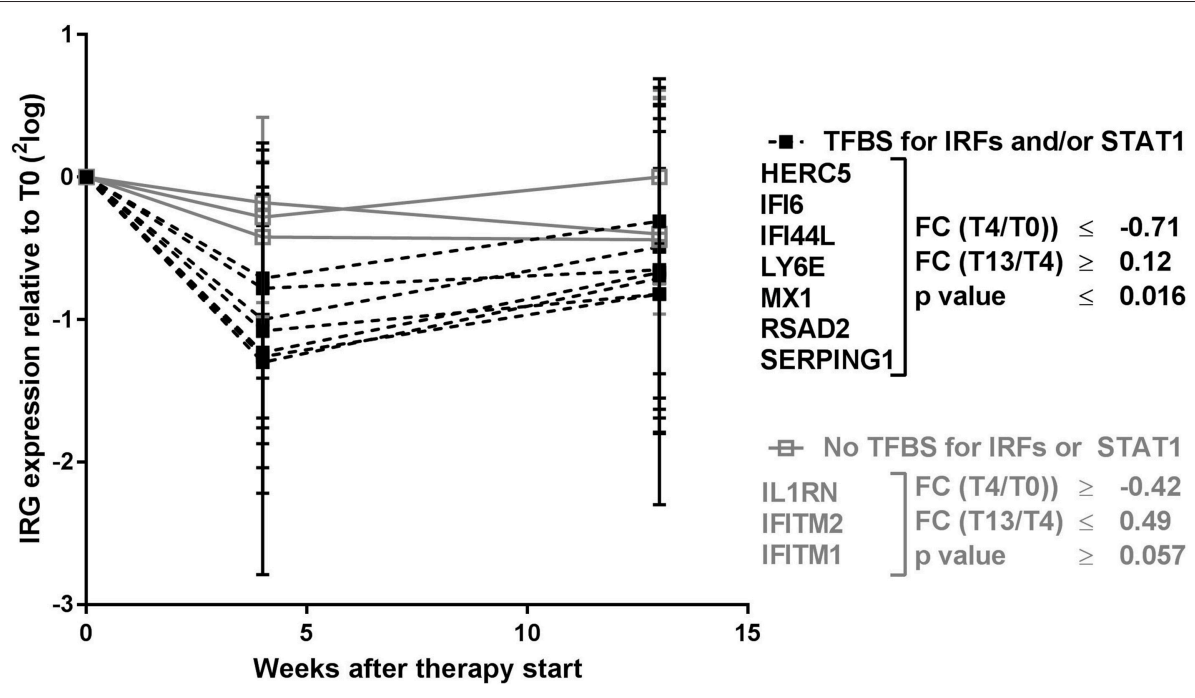

FIGURE 2 | Expression dynamics of individual IRGs during COBRA and COBRA-light therapy. IRGs were categorized based on the presence or absence of transcription factor binding sites (TFBS) for IRF proteins and/or STAT1. FC, fold change expressed in ${ }^{2}$ log values. P-values are indicated for longitudinal analysis by Friedman test.

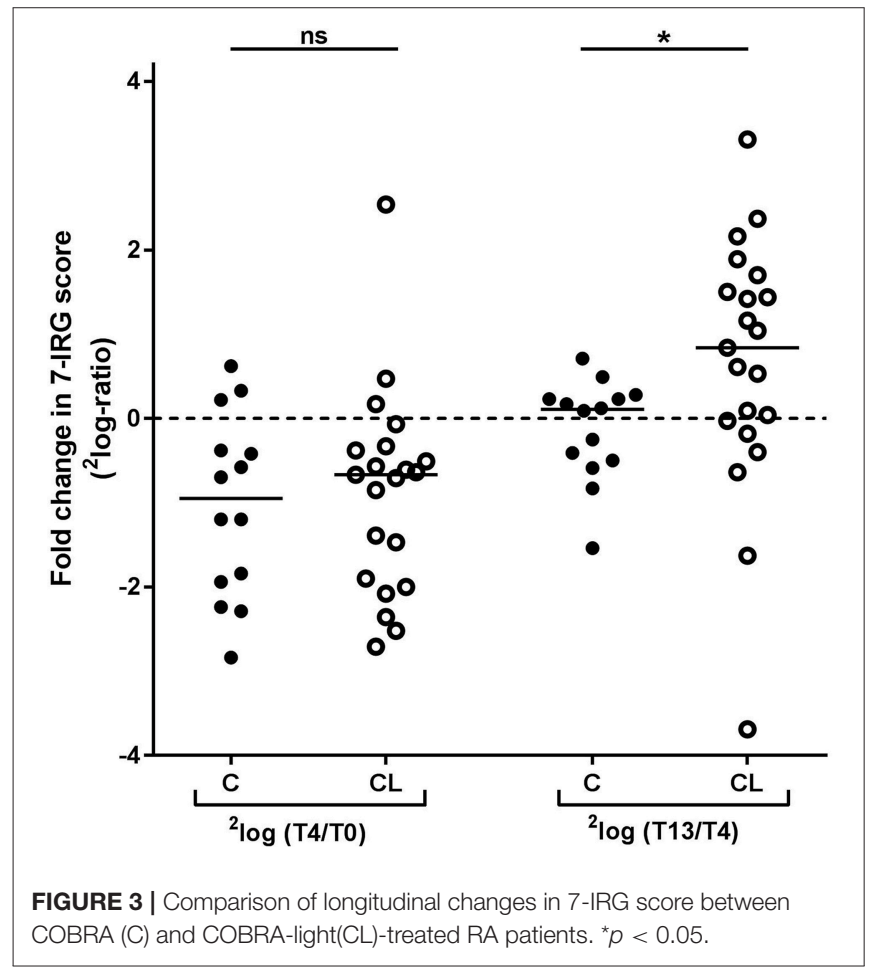

and later time points as indicators of inflammation. Interestingly, a significant positive correlation was observed between the change in IRG expression and change in both CRP and ESR between T0 and T4 ( $p \leq 0.051$, Pearson $r \geq 0.42$ for 7-IRG score, see Table 3 and see Supplementary Tables 4, 5 for the individual IRGs). However, this correlation was diminished at later time points, suggesting that there is no relation with the eventual
TABLE 3 | Assessment of 7-IRG score values and dynamics in relation to clinical response to COBRA and COBRA-light therapy.

\begin{tabular}{|c|c|c|c|c|c|}
\hline & \multicolumn{3}{|c|}{$\begin{array}{l}\text { 7-IRG score at } \\
\text { time point }\end{array}$} & \multicolumn{2}{|c|}{$\begin{array}{c}{ }^{2} \text { log-ratios in 7-IRG } \\
\text { score }\end{array}$} \\
\hline & то & T4 & T13 & $\mathrm{T} 4 / \mathrm{TO}$ & $\mathrm{T} 13 / \mathrm{T} 4$ \\
\hline $\begin{array}{l}\text { R vs. NR } \\
\text { (DAS } \leq 2.4 \text { or }>2.4 \text { at T26) }\end{array}$ & 0.17 & 0.23 & 0.56 & 0.86 & 0.53 \\
\hline$\triangle \mathrm{DAS}$ at $\mathrm{T} 13$ (correlation) & 0.18 & 0.21 & 0.72 & 0.43 & 0.29 \\
\hline$\triangle \mathrm{DAS}$ at T26 (correlation) & 0.70 & 0.32 & 0.56 & 0.58 & 0.93 \\
\hline${ }^{2}$ Log-ratio CRP (T4/T0) & 0.31 & 0.34 & 0.81 & $0.010(+)$ & 0.22 \\
\hline${ }^{2}$ Log-ratio CRP (T13/T0) & 0.087 & 0.68 & 0.54 & 0.066 & 0.25 \\
\hline${ }^{2}$ Log-ratio CRP (T26/T0) & 0.12 & 0.17 & 0.30 & 0.61 & 0.90 \\
\hline${ }^{2}$ Log-ratio ESR (T4/TO) & 0.23 & 0.49 & 0.68 & $0.013(+)$ & 0.84 \\
\hline${ }^{2}$ Log-ratio ESR (T13/T0) & 0.083 & 0.85 & 0.55 & $0.038(+)$ & 0.36 \\
\hline${ }^{2}$ Log-ratio ESR (T26/T0) & 0.063 & 0.36 & 0.63 & 0.16 & 0.75 \\
\hline
\end{tabular}

Table indicates $p$ values. Details of the statistical analyses are described in the methods section. The direction of the significant correlations is indicated between brackets.

clinical response to COBRA and COBRA-light therapy. Separate analysis of the COBRA and COBRA-light group revealed similar results (data not shown).

\section{DISCUSSION}

In previous studies using cross-sectional data from RA patients, we observed lower IRG expression in patients using GCs, SSZ and hydroxychloroquine, but not in patients using MTX (7, 15). The unique and virtually complete longitudinal collection of PAXgene blood enabled us to investigate the influence of immunosuppressive therapy on the IFN response in a 
longitudinal setting. To our knowledge, the present study is the first to do so.

Using blood collected at baseline and after 4 and 13 weeks of COBRA or COBRA-light treatment, we observed a substantial downregulation of the IFN response within 4 weeks of therapy. This reduction was irrespective of the therapy group, but was not equally strong for each IRG. Between 4 and 13 weeks, however, IRG expression changes were highly variable between patients, which appeared partly dependent on the treatment.

The extent of the downregulation after 4 weeks of treatment was similar between COBRA and COBRA-light-treated patients. Most probably, this decline is due to the prednisolone treatment, as its dose is relatively high in both groups, and it acts more rapidly than MTX and SSZ. The absence of differences between COBRA and COBRA-light treatment at this time point suggests that prednisolone dose of $30 \mathrm{mg} /$ day prednisolone already causes maximum downregulation. The expression dynamics seemed to be restricted to IRGs that contained one or more binding sites for IRF transcription factors and/or STAT1. Conversely, three genes that lacked such binding sites, displayed considerably less downregulation during treatment. Previous in vitro studies have shown that the GC signaling pathway, which is activated by prednisolone, is able to compete with the IFN signaling pathway for certain IRF proteins $(12,13)$ and to inhibit STAT1 activation (14), which could explain our observations.

Between T4 and T13, the IRG dynamics were more variable and differed between the two patient groups. Whereas normalization of IRG expression toward baseline levels was observed in the COBRA-light-treated group, IRG expression remained rather stable in the COBRA-treated group. This is particularly remarkable, as the prednisolone dose is equal between both groups at after 12 weeks $(7.5 \mathrm{mg} /$ day $)$, and the only difference is the MTX dose $(7.5 \mathrm{mg} /$ week in COBRA and $25 \mathrm{mg} /$ week in COBRA-light) and the addition of $2 \mathrm{~g}$ SSZ in the COBRA-treated groups. The total received dose of prednisolone, however, is 1.5-fold higher in the COBRAtreated group at this point. Possibly, the combination of SSZ and higher total prednisolone dose causes a more prolonged downregulation of the IFN response in the COBRA group. However, due to the combination of agents, it is not possible to strongly conclude which agent is responsible for the observed differences in dynamics.

Unfortunately, no untreated control-group with longitudinal follow-up was available, hence it cannot be fully excluded that the IRG dynamics we observed were a consequence of natural fluctuation. However, the correspondence with previously published in vitro data $(12,13)$ as well as our previous in vivo data $(7,15)$ and the observed differences between COBRA and COBRA-light strongly suggest that the observed changes in IRG expression are not spontaneous but truly mediated by the treatment.

The observation that not all IRGs appeared equally sensitive to the immunosuppressive agents of COBRA and COBRA-light therapies, and the putative influence of total prednisolone dose, could particularly be important when using the IFN response as a biomarker, which has been described for several biologics, including TNF inhibitors, rituximab, and tocilizumab $(8,10,11$,
19, 20). For example, we have demonstrated that the predictive performance of the 8-IRG geneset for non-response to rituximab is reduced when patients use prednisolone at the moment of blood collection, presumably because of a prednisolonemediated reduction in IRG expression (7). Correspondingly, for 5 of the 8 genes in this geneset we have now shown that they indeed are sensitive to immunosuppressive treatment, including prednisolone.

Remarkably, the observation that the IRG downregulation attenuated in COBRA-light-treated patients implies that the IFN response could normalize upon reduction of the prednisolone dose. Hence, the 8-IRG geneset might still be applicable as a predictor for rituximab in patients who are tapering their prednisolone.

Moreover, it would be particularly interesting to investigate whether the IRGs that were less affected by COBRA and COBRAlight treatment could serve as alternative predictors for the response to biologics, since they do reflect IFN activity in RA (17), hence they might still play a role in the response to biologics. Interestingly, the gene IFITM1, which appeared less sensitive to prednisolone interference, has already been described as a predictor of rituximab nonresponse in a transcriptomics study (21). Alternatively, one study demonstrated an association between IFN-related gene variants and the response to rituximab (22). Although the predictive value was rather low, the concept of using IFN-related gene variants, which are naturally insensitive to therapy interference, would be interesting to study in further detail and with more IFN-related SNPs (23).

Besides the differential sensitivities of individual IRGs to the treatments, we also observed high heterogeneity in the IRG dynamics between patients. As described before, IRG expression in RA patients is generally highly heterogeneous, which we observed both at baseline and upon therapy. Although we observed a linear relation between baseline IRG expression and the extent of the downregulation after 4 weeks, the variation in IRG dynamics could not be fully explained by the baseline variation in IRG expression. This indicates that besides the type of treatment and the administered doses of treatment, there are also other factors that could influence the IFN response in RA. It has been well-discussed that the IRG response in RA patients is the result of several factors combined, such as extracellular stimuli (24), receptor expression (25) and genetic variation in signaling proteins $(22,23,26)$. Considering the putative mechanism of IRG downregulation by prednisolone as described above, particularly the variation in signaling proteins could also contribute to a patient's sensitivity to the observed IRG downregulation. In addition, many other factors, independent of baseline IRG expression, such as therapy adherence and the patient's sensitivity to glucocorticoids (27) could hypothetically affect the extent of the IRG downregulation.

Despite this heterogeneity in the IFN response between patients, we did not observe an association between the IRG expression or dynamics and the response to COBRA and COBRA-light therapy. Considering the differences in IRG dynamics between COBRA and COBRA-light, the potential relation between IRG expression and clinical response should ideally be analyzed for both treatment groups separately. 
Since methotrexate has no proven interference with IRG expression, while prednisolone and sulfasalazine have, the use of IRG-interfering agents is considerably higher with COBRAtreatment compared to COBRA-light treatment. Moreover, as all agents have different modes of action (28-30), hence clinical response for each agent is probably achieved via different mechanisms. Consequently, it is possible that the relation between IRG expression and clinical response is different between COBRA and COBRA-light. Unfortunately, the current cohorts were too small to study this in detail.

Since DAS information was not available at T4, a direct comparison of DAS dynamics and IRG dynamics could not be made. Instead, we additionally investigated CRP and ESR as indicators of changes in inflammation in relation to IRG dynamics. Interestingly, a significant correlation was observed between IRG decline and CRP and ESR decline at T4, but not at later time points. At this early time point, clinical effects are mostly attributed to the prednisolone treatment, whereas at later time points more influence is anticipated from MTX and SSZ. As a consequence, the IRG dynamics at T4 could reflect the initial clinical response to prednisolone, but it does not predict the eventual clinical response as this is the result of the combination of agents. It would be interesting to study the potential relation between IRG dynamics and clinical response in patients using prednisone as monotherapy compared to patients using MTX and/or SSZ monotherapy.

In summary, we have demonstrated that both COBRA and COBRA-light therapy are able to downregulate the IFN response in RA. The dynamics of this downregulation were partly dependent on the presence of TFBS within the IRGs and the combination and dosages of agents, but they were irrespective of the clinical response to therapy. Altogether, these results shed a new light on the behavior of the IFN response in RA.

\section{REFERENCES}

1. Lard LR, Visser H, Speyer I, vander Horst-Bruinsma IE, Zwinderman $\mathrm{AH}$, Breedveld FC, et al. Early versus delayed treatment in patients with recent-onset rheumatoid arthritis: comparison of two cohorts who received different treatment strategies. Am J Med. (2001) 111:446-51. doi: 10.1016/S0002-9343(01)00872-5

2. van Aken J, Lard LR, le Cessie S, Hazes JM, Breedveld FC, Huizinga TW. Radiological outcome after four years of early versus delayed treatment strategy in patients with recent onset rheumatoid arthritis. Ann Rheum Dis. (2004) 63:274-9. doi: 10.1136/ard.2003.010298

3. den Uyl D, ter Wee M, Boers M, Kerstens P, Voskuyl A, Nurmohamed M, et al. A non-inferiority trial of an attenuated combination strategy ('COBRA-light') compared to the original COBRA strategy: clinical results after 26 weeks. Ann Rheum Dis. (2014) 73:1071-8. doi: 10.1136/annrheumdis-2012-202818

4. ter Wee MM, den Uyl D, Boers M, Kerstens P, Nurmohamed M, van Schaardenburg D, et al. Intensive combination treatment regimens, including prednisolone, are effective in treating patients with early rheumatoid arthritis regardless of additional etanercept: 1-year results of the COBRA-light openlabel, randomised, non-inferiority trial. Ann Rheum Dis. (2015) 74:1233-40. doi: 10.1136/annrheumdis-2013-205143

5. Konijn NPC, van Tuyl LHD, Boers M, den Uyl D, Ter Wee MM, van der Wijden LKM, et al. Similar efficacy and safety of initial COBRA-light and COBRA therapy in rheumatoid arthritis: 4-year results from the COBRA-light trial. Rheumatology. (2017) 56:1586-96. doi: 10.1093/rheumatology/kex223

\section{ETHICS STATEMENT}

This study was approved by the medical ethics committee of VU University Medical Center and Reade, Amsterdam, The Netherlands, and informed consent was obtained from all donors.

\section{AUTHOR CONTRIBUTIONS}

All authors were involved in drafting the article or revising it critically for important intellectual content, and all authors approved the final version to be published. TdJ and WL: study concept and design. TdJ, TS, EM, and WL: acquisition of patient material and data. TdJ, TS, EM, CvdL, RvV, and WL: analysis and interpretation of data.

\section{FUNDING}

This study was performed with support from the Dutch Arthritis Foundation (project number LLP-20). The COBRA-light trial was supported by an unrestricted grant from Pfizer.

\section{ACKNOWLEDGMENTS}

The authors thank Ittai Muller for his contribution to the sample acquisition and processing and Linda Rasch for the clinical data acquisition.

\section{SUPPLEMENTARY MATERIAL}

The Supplementary Material for this article can be found online at: https://www.frontiersin.org/articles/10.3389/fimmu. 2019.00902/full\#supplementary-material

6. Smolen JS, Landewe R, Bijlsma J, Burmester G, Chatzidionysiou K, Dougados $\mathrm{M}$, et al. EULAR recommendations for the management of rheumatoid arthritis with synthetic and biological disease-modifying antirheumatic drugs: 2016 update. Ann Rheum Dis. (2017) 76:960-77. doi: 10.1136/annrheumdis-2016-210715

7. de Jong TD, Vosslamber S, Blits M, Wolbink G, Nurmohamed MT, van der Laken CJ, et al. Effect of prednisone on type I interferon signature in rheumatoid arthritis: consequences for response prediction to rituximab. Arthr Res Ther. (2015) 17:78. doi: 10.1186/s13075-015-0 564-y

8. Thurlings RM, Boumans M, Tekstra J, van Roon JA, Vos K, van Westing DM, et al. Relationship between the type I interferon signature and the response to rituximab in rheumatoid arthritis patients. Arthr Rheum. (2010) 62:3607-14. doi: 10.1002/art.27702

9. Lubbers J, Brink M, van de Stadt LA, Vosslamber S, Wesseling JG, van Schaardenburg D, et al. The type I IFN signature as a biomarker of preclinical rheumatoid arthritis. Ann Rheum Dis. (2013) 72:776-80. doi: 10.1136/annrheumdis-2012-202753

10. Sanayama Y, Ikeda K, Saito Y, Kagami S, Yamagata M, Furuta S, et al. Prediction of therapeutic responses to tocilizumab in patients with rheumatoid arthritis: biomarkers identified by analysis of gene expression in peripheral blood mononuclear cells using genome-wide DNA microarray. Arthr Rheum. (2014) 66:1421-31. doi: 10.1002/art.38400

11. Wright HL, Thomas HB, Moots RJ, Edwards SW. Interferon gene expression signature in rheumatoid arthritis neutrophils correlates with 
a good response to TNFi therapy. Rheumatology. (2015) 54:188-93. doi: 10.1093/rheumatology/keu299

12. Reily MM, Pantoja C, Hu X, Chinenov Y, Rogatsky I. The GRIP1:IRF3 interaction as a target for glucocorticoid receptormediated immunosuppression. EMBO J. (2006) 25:108-17. doi: 10.1038/sj.emboj.7600919

13. Flammer JR, Dobrovolna J, Kennedy MA, Chinenov Y, Glass CK, Ivashkiv LB, et al. The type I interferon signaling pathway is a target for glucocorticoid inhibition. Mol Cell Biol. (2010) 30:4564-74. doi: 10.1128/MCB. 00146-10

14. Bhattacharyya S, Zhao Y, Kay TW, Muglia LJ. Glucocorticoids target suppressor of cytokine signaling 1 (SOCS1) and type 1 interferons to regulate Toll-like receptor-induced STAT1 activation. Proc Natl Acad Sci USA. (2011) 108:9554-9. doi: 10.1073/pnas.1017296108

15. de Jong TD, Blits M, de Ridder S, Vosslamber S, Wolbink G, Nurmohamed MT, et al. Type I interferon response gene expression in established rheumatoid arthritis is not associated with clinical parameters. Arthr Res Ther. (2016) 18:290. doi: 10.1186/s13075-016-1191-y

16. Arnett FC, Edworthy SM, Bloch DA, McShane DJ, Fries JF, Cooper NS, et al. The American Rheumatism Association 1987 revised criteria for the classification of rheumatoid arthritis. Arthritis Rheum. (1988) 31:315-24.

17. van der Pouw Kraan TC, Wijbrandts CA, van Baarsen LG, Voskuyl AE, Rustenburg F, Baggen JM, et al. Rheumatoid arthritis subtypes identified by genomic profiling of peripheral blood cells: assignment of a type I interferon signature in a subpopulation of patients. Ann Rheum Dis. (2007) 66:1008-14. doi: 10.1136/ard.2006.063412

18. Rusinova I, Forster S, Yu S, Kannan A, Masse M, Cumming H, et al. Interferome v2.0: an updated database of annotated interferonregulated genes. Nucleic acids Res. (2013) 41(Database issue):D1040-6. doi: $10.1093 / \mathrm{nar} / \mathrm{gks} 1215$

19. van Baarsen LG, Wijbrandts CA, Rustenburg F, Cantaert T, van der Pouw Kraan TC, Baeten DL, et al. Regulation of IFN response gene activity during infliximab treatment in rheumatoid arthritis is associated with clinical response to treatment. Arthr Res Ther. (2010) 12:R11. doi: 10.1186/a $\mathrm{r} 2912$

20. Raterman HG, Vosslamber S, de Ridder S, Nurmohamed MT, Lems WF, Boers $\mathrm{M}$, et al. The interferon type I signature towards prediction of non-response to rituximab in rheumatoid arthritis patients. Arthr Res Ther. (2012) 14:R95. doi: $10.1186 / \mathrm{ar} 3819$

21. Sellam J, Marion-Thore S, Dumont F, Jacques S, Garchon HJ, Rouanet $S$, et al. Use of whole-blood transcriptomic profiling to highlight several pathophysiologic pathways associated with response to rituximab in patients with rheumatoid arthritis: data from a randomized, controlled, open-label trial. Arthr Res Ther. (2014) 66:2015-25. doi: 10.1002/art. 38671

22. Juge PA, Gazal S, Constantin A, Mariette X, Combe B, Tebib J, et al. Variants of genes implicated in type 1 interferon pathway and B-cell activation modulate the EULAR response to rituximab at 24 weeks in rheumatoid arthritis. $R M D$ Open. (2017) 3:e000448. doi: 10.1136/rmdopen-2017-000448

23. Delgado-Vega AM, Alarcon-Riquelme ME, Kozyrev SV. Genetic associations in type I interferon related pathways with autoimmunity. Arthr Res Ther. (2010) 12(Suppl. 1):S2. doi: 10.1186/ar2883

24. Baccala R, Hoebe K, Kono DH, Beutler B, Theofilopoulos AN. TLR-dependent and TLR-independent pathways of type I interferon induction in systemic autoimmunity. Nat Med. (2005) 13:543-51. doi: 10.1038/nm1590

25. de Jong TD, Lübbers J, Turk S, Vosslamber S, Mantel E, Bontkes HJ, et al. The type I interferon signature in leukocyte subsets from peripheral blood of patients with early arthritis: a major contribution by granulocytes. Arthr Res Ther. (2016) 18:165. doi: 10.1186/s13075-016-1065-3

26. Okada Y, Wu D, Trynka G, Raj T, Terao C, Ikari K, et al. Genetics of rheumatoid arthritis contributes to biology and drug discovery. Nature. (2014) 506:376-81. doi: 10.1038/nature12873

27. Quax RA, Koper JW, de Jong PH, van Heerebeek R, Weel AE, Huisman AM, et al. In vitro glucocorticoid sensitivity is associated with clinical glucocorticoid therapy outcome in rheumatoid arthritis. Arthr Res Ther. (2012) 14:R195. doi: $10.1186 /$ ar4029

28. Tian H, Cronstein BN. Understanding the mechanisms of action of methotrexate: implications for the treatment of rheumatoid arthritis. Bulletin of the NYU hospital for joint diseases. Bull NYU Hosp Jt Dis. (2007) 65:168-73. Available online at: http://hjdbulletin.org/files/archive/pdfs/532.pdf

29. Rhen T, Cidlowski JA. Antiinflammatory action of glucocorticoidsnew mechanisms for old drugs. N. Engl. J Med. (2005) 353:1711-23. doi: 10.1056/NEJMra050541

30. Smedegard G, Bjork J. Sulphasalazine: mechanism of action in rheumatoid arthritis. $\operatorname{Br} \quad J$ Rheumatol. (1995) 34(Suppl. 2):7-15. doi: 10.1093/rheumatology/XXXIV.suppl_2.7

Conflict of Interest Statement: RvV reports grants and research support from AbbVie, Amgen, Bristol-Myers Squibb, GSK, Lilly, Pfizer, Roche, and UCB Pharma and personal fees from AbbVie, Biotest, Bristol-Myers Squibb, Celgene, Crescendo, GSK, Janssen, Lilly, Novartis, Pfizer, Roche, and UCB pharma, outside the submitted work.

The remaining authors declare that the research was conducted in the absence of any commercial or financial relationships that could be construed as a potential conflict of interest.

Copyright (c) 2019 de Jong, Snoek, Mantel, van der Laken, van Vollenhoven and Lems. This is an open-access article distributed under the terms of the Creative Commons Attribution License (CC BY). The use, distribution or reproduction in other forums is permitted, provided the original author(s) and the copyright owner(s) are credited and that the original publication in this journal is cited, in accordance with accepted academic practice. No use, distribution or reproduction is permitted which does not comply with these terms. 